\title{
Preparation of Pectin Films from Coffee Cherry and Its Antibacterial Activity
}

\author{
Sunita Chamyuang ${ }^{1,2, *}$, Sitthi Duangphet ${ }^{1,3}$, Amorn Owatworakit ${ }^{1,2}$, \\ Uraiwan Intatha ${ }^{1,3}$, Jutamat Nacha ${ }^{2}$ and Panalee Kerdthong ${ }^{1}$ \\ ${ }^{1}$ School of Science, Mae Fah Luang University, Chiang Rai 57100, Thailand \\ ${ }^{2}$ Microbial Products and Innovation Research Group, Mae Fah Luang University, \\ Chiang Rai 57100, Thailand \\ ${ }^{3}$ Center of Innovative Materials for Sustainability (iMatS), Mae Fah Luang University, \\ Chiang Rai 57100, Thailand
}

\section{("Corresponding author's e-mail: sunita@mfu.ac.th)}

Received: 9 June 2020, Revised: 9 April 2021, Accepted: 19 April 2021

\begin{abstract}
This study aimed to utilize the waste from the coffee bean production process as high value-added material. Pectin extracted from the coffee pulp was classified as low methoxyl pectin (LMP) with the Degree of Esterification (DE) at $33.33 \%$ For further application or upscaling production, the coffee pulp was boiled to retrieve the highest yield at $15.68 \mathrm{~g} / 100 \mathrm{~g} \mathrm{DW}$. The antimicrobial assays of crude pectin via minimum inhibition concentration (MIC) showed that coffee pectin could inhibit the growth of Staphylococcus aureus TISTR 1466 in the range of $2.5-0.62 \mathrm{mg}$, while the commercial apple pectin exhibited the inhibition only slightly. The pectin film from the coffee pectin blended with the commercial apple pectin also exhibited antimicrobial activity against $S$. aureus TISTR 1466 . This study shows that coffee pectin could be an alternative material for antibacterial film and other applications.
\end{abstract}

Keywords: Coffee cherry pectin, Low methoxyl pectin (LMP), Antibacterial activity, Pectin film

\section{Introduction}

Arabica coffee production in Thailand is around 12,000 tons per year. Of these, 4,900 tons are produced in Chiang Rai province, the largest producer of Arabica coffee in Thailand [1]. Forty-five percent of the harvested coffee cherries, which are green coffee beans, undergo coffee roasting. The other $55 \%$ is agricultural waste, composed mainly of skin, pulp, mucilage and parchment. Coffee waste is commonly used as fertilizer or animal feed [2]. Coffee pulp is an agricultural commodity that can be used for other applications, especially as an alternative fiber source or materials. It comprises carbohydrates, including pectin (20 - 35\%), oligosaccharides and fiber (30\%), protein, minerals, and water. It has been estimated that $10 \mathrm{~kg}$ of the coffee pulp can yield up to $3.5 \mathrm{~kg}$ of pectin $[3,4]$.

Pectin is a natural polysaccharide found in plant cell walls, and is extensively used in the food industry as gelling, stabilizing, and thickening agent. Chemically, pectin is a polymer made of Dgalacturonic acid (GalA) monomers linked to each other by $\alpha-(1 \rightarrow 4)$ galacturonosyl linkages. Part of the carboxyl groups of GalA is esterified with methoxyl groups (as shown in Figure 1), while structure varies according to degree of esterification (DE). Thus, pectins are classified as high methoxyl pectins (HMPs) with $\mathrm{DE}>50 \%$ and low methoxyl pectin (LMPs) with $\mathrm{DE}<50 \%$ [5]. Due to differences in the substituted groups, the gelation mechanism of HMP and LMP also differ. The HMPs form gel at low $\mathrm{pH}$ $(<3.5)$ in the presence of sucrose, while LMPs form gel at a wide range of $\mathrm{pH}$ in the presence of calcium ions $(\mathrm{Ca} 2+)$. The composition and structure of pectin depend on the source of the plant and extraction conditions [6]. 


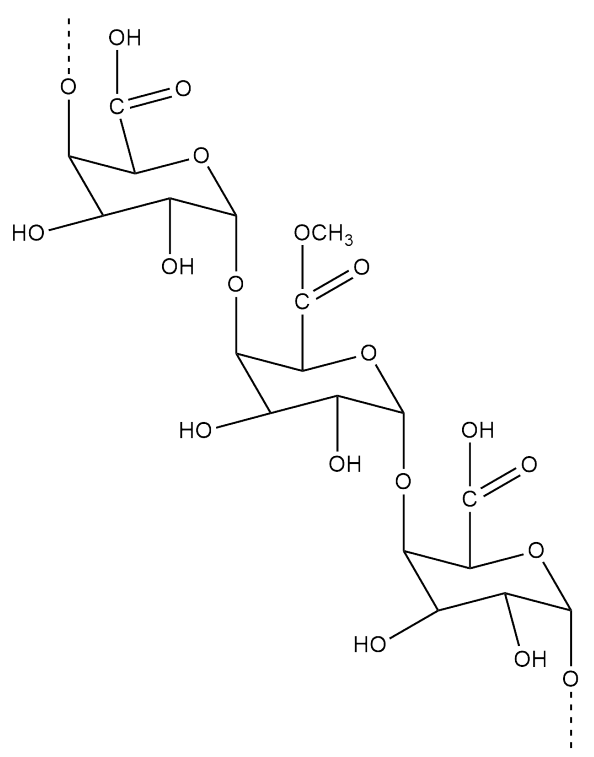

Figure 1 Structure of pectin.

Due to the different gelation mechanisms of HMPs and LMPs, the utilization of these 2 pectins also varies. For instance, HMPs are commonly used in the food industry as stabilizers and thickening agents, while LMPs are used in biomedical and pharmaceutical applications, such as drug-controlled release matrices, wound healing agents, glucose-lowering agents and alternative fiber prebiotics [7]. Commercial pectins are mainly produced from citrus peels and apple pomace, both of which yield HMPs. Despite the general used as a gelling agent in jams and jellies and as a stabilizing agent in fruit juice and dairy products, it is also used as dietary fiber. In the previous study, the antimicrobial activity of pectin alone was not extensively reported in the literature. However, a report that used citrus pectin in the functional films showed inhibitory effects against L. monocytogenes, E. coli and S. aureus [8,9]. Furthermore, Daoud et al. [10] reported that the lyophilized citrus pectin showed activities against 16 clinical isolates and 2 reference strains of Helicobacter pylori. Nevertheless, the antimicrobial activities were reported from HMP from citrus but not LMP from coffee pectin. Previously, Garcia et al. [3] extracted LMP pectin from coffee pulps using hydrochloric acid as solven. Therefore, the present work aims to extract pectin from Arabica coffee by using citric acid as an organic solvent and employing different extraction methods, including boiling, microwave-assisted, and autoclave-assisted. Yield and chemical properties of pectin from each extraction were compared. Pectin-based antibacterial films were also prepared, and their antibacterial properties were examined.

\section{Materials and methods}

Materials

Coffee cherry pulp (Coffea arabica var. Catimor) was supplied by Akha Coffee Co. Ltd, from a coffee plantation in Mae Suai district, Chiang Rai province, Thailand, during the 2018 harvest. The coffee cherry was stored at $-20^{\circ} \mathrm{C}$ prior to extraction.

\section{Pectin extraction method}

The extraction method was optimized from Rakitikul et al. [11] Coffee cherry pulp was macerated with $1 \mathrm{M}$ citric acid in a ratio of $1: 4$ (coffee cherry pulp: citric acid) using $\mathrm{T} 50$ digital ULTRATURRAX ${ }^{\circledR}$ at $8,000 \mathrm{rpm}$ for $10 \mathrm{~min}$. An equal amount of slurry was separated into 3 portions for different extraction treatments. The first portion was heated at $90{ }^{\circ} \mathrm{C}$ with continuous stirring at $180 \mathrm{rpm}$ for $90 \mathrm{~min}$. The second portion was subjected to microwave-assisted extracting method using a microwave at $900 \mathrm{~W}$ for $15 \mathrm{~min}$. The last portion was extracted by autoclave under the condition of 121 ${ }^{\circ} \mathrm{C}, 15 \mathrm{psi}$ for $15 \mathrm{~min}$. Slurry from each treatment was cooled down to $45^{\circ} \mathrm{C}$, filtered with a cheesecloth, and the filtrates were adjusted at $\mathrm{pH}$ of 5.0 with sodium hydroxide $(\mathrm{NaOH})$. One volume of filtrate was precipitated with 2 volumes of $95 \%$ ethanol for $6 \mathrm{~h}$ at room temperature. Each suspension was then filtrated again through 400-micron nylon mesh yielding crude coffee pectin on top of the mesh. These crude pectins were washed 3 times with $70 \%$ ethanol and dried at $60{ }^{\circ} \mathrm{C}$ for $12 \mathrm{~h}$. The dried coffee pectin 
from each treatment was ground to a fine powder and kept in a desiccator for further study. Each extraction treatment was performed in triplicate. The pectin yield was calculated by means of Eq. (1);

Pectin yield $(\%)=\frac{\text { Weight of product obtained }(g)}{\text { Weight of coffee pulp }(g)} \times 100$

Fourier Transform Infrared (FTIR) spectroscopy assay

Commercial apple pectin and crude coffee pectin were subjected to FTIR spectroscopy using a Perkin Elmer FTIR spectrometer (Perkin Elmer co., MA, USA). Samples were ground into a fine powder using mortar and grinder prior to compression into $\mathrm{KBr}$ discs. The characteristic spectra were recorded in the range $4000-450 \mathrm{~cm}^{-1}$ at a resolution of $4 \mathrm{~cm}^{-1}$ [12].

\section{Determination of Degree of Esterification (DE) of pectin}

The degree of esterification (DE) of pectin was determined by the titrimetric method adapted from Dranca et al. [13]. The analyses were performed in triplicate; $0.2 \mathrm{~g}$ of dried crude pectin were solubilized in $20 \mathrm{~mL}$ of distilled water at $40{ }^{\circ} \mathrm{C}$. After the samples were completely dissolved, 5 drops of phenolphthalein were added to the samples and titrated with $0.1 \mathrm{~N}$ sodium hydroxide $(\mathrm{NaOH})$; the volume of used $\mathrm{NaOH}$ was recorded as V1. Subsequently, $10 \mathrm{~mL}$ of $0.1 \mathrm{~N} \mathrm{NaOH}$ were added and the solution was stirred for $15 \mathrm{~min}$, followed by the addition of $10 \mathrm{~mL}$ of $0.1 \mathrm{~N}$ hydrochloric acid $(\mathrm{HCl})$, while vigorously stirring (until disappearance of pink color). The final titration was made with $0.1 \mathrm{~N}$ $\mathrm{NaOH}$. The end of titration was signified when the solution turned pink. The volume of $\mathrm{NaOH}$ was recorded as V2. The degree of esterification was calculated using Eq. (2);

Degree of Esterification $(\%)=\frac{V_{2}}{V_{1}+V_{2}} \times 100$

\section{Thermal analysis}

The thermal decomposition behavior of the blends was investigated with a TGA SDTA 851e (Mettler Toledo, USA) using a temperature that ranged from $25-1,400{ }^{\circ} \mathrm{C}$ with a heating rate of 10 ${ }^{\circ} \mathrm{C} / \mathrm{min}$. Approximately $20 \mathrm{mg}$ of sample was used.

\section{Preparation of antibacterial films from coffee pectin}

Antibacterial films were prepared by the solvent casting method using commercial apple pectin as a matrix. Commercial apple pectin was dissolved in deionized water to the optimum concentration of $5 \%$ w/v. After the solution was homogenous, the pectin extracted from the coffee pulp was added in various concentrations; $0,1,3$ and $5 \% \mathrm{w} / \mathrm{w}$ of solid content. The mixtures were sonicated to remove bubbles and then casted into a silicone mold. The solution was allowed to dry in the oven at $30^{\circ} \mathrm{C}$ for $48 \mathrm{~h}$. The film from each condition was cut into a circular film with $7 \mathrm{~mm}$ diameter and brought to autoclave of $121{ }^{\circ} \mathrm{C}$, 15 psi for 15 min prior to the antimicrobial study.

\section{Determination of the minimum inhibition concentration (MIC) of crude coffee pectin}

The MIC was determined in Mueller-Hinton Broth (MHB) from HIMEDIA, India, according to the guidelines of the CLSI microdilution method and adapted from Roser et al. [14] and Balouiri et al. [15]. The experiments were done on a 96-well plate. In brief, $200 \mu \mathrm{L}$ MHB was added. In the first column, coffee pectin or commercial apple pectin was added to a $10 \mathrm{mg} / \mathrm{mL}$ final concentration. Then, 1 -fold serial dilutions were performed sequentially from the first column to the next until a final concentration of $0.15 \mathrm{mg} / \mathrm{mL}$ was reached. Subsequently, $20 \mu \mathrm{L}$ of Staphylococcus aureus TISTR 1466 suspension at 0.05 O.D. at $660 \mathrm{~nm}$ were added into each well except for the blank well, which was set as the blank media. A well containing HMB with culture only and another containing HMB with Gentamicin and culture were used as control. Experiments were repeated in 3 replicates.

The growth of the bacteria was determined by turbidimetric determination using a microplate reader at O.D. $660 \mathrm{~nm}$ reading at $0,2,4,6,8$ and $12 \mathrm{~h}$ intervals.

\section{Antimicrobial properties of coffee pectin film}

Antibacterial activity of the coffee pectin film was determined using the Kirby-Bauer disk diffusion method [16]. Briefly, a culture of Staphylococcus aureus TISTR 1466 was inoculated into a sterile nutrient broth and incubated at $37{ }^{\circ} \mathrm{C}$ on a rotary shaker at $150 \mathrm{rpm}$. The bacterial culture was grown until it reached $0.5 \mathrm{McF}$ arland, at which point it was transferred on a nutrient agar plate. A $0.7 \mathrm{~mm}$ diameter pectin disc was placed on the agar plate with bacterial lawn. Gentamicin $(10 \mu \mathrm{g})$ susceptibility discs from 
Oxoid were used as standard. The anti-microbial activity was expressed as zone of inhibition in millimeters. This was measured by a ruler after the plates were incubated at $37{ }^{\circ} \mathrm{C}$ for $24 \mathrm{~h}$. Each treatment of pectin film was conducted in triplicate.

\section{Statistical analysis}

All data were presented in mean $\pm \mathrm{SD}$. The statistical analysis was performed by 2-way analysis of variance (ANOVA), 1-way analysis of variance (ANOVA), independent t-test and Duncan's posttest. $p$-value less than 0.05 were examined significant. Statistical Package for the Social Sciences (SPSS) version no. 25 was used to analysis statistic in this work.

\section{Results and discussion}

Yield and Degree of Esterification (DE) of pectin

Coffee pulps were extracted with 3 different extraction methods; boiling, microwave-assisted, and autoclave-assisted as stated in section 4.2. Each extraction method yielded pale brown pectin powder. Percent yield (g pectin/100 g dry weight of coffee pulp) and \%DE of crude coffee pectin from each extraction method and commercial pectin from apple pulp are summarized in Table $\mathbf{1 .}$

Table 1 The yield of pectin (g/100 g dried weight) using 3 extraction methods and degree of esterification of each pectin sample.

\begin{tabular}{ccc}
\hline Pectin sample & \% Yield & \% DE \\
\hline Boiling & $15.9 \pm 0.4$ & $28.6 \pm 0.2$ \\
Microwave assisted & $9.3 \pm 0.6$ & $44.4 \pm 0.1$ \\
Autoclave assisted & $10.7 \pm 03$ & $50.2 \pm 0.1$ \\
Commercial & $\mathrm{n} / \mathrm{a}$ & $66.67 \pm 0.2$ \\
\hline
\end{tabular}

The results indicated that the coffee pectin from all extraction methods was of the low methoxyl type. Nonetheless, the boiling extraction method yielded the most pectin, which was still $4.32 \%$ lower than the maximum estimated pectin in coffee pulp (35\%).

\section{Fourier Transform Infrared (FTIR) spectroscopy assay}

FTIR spectra identified pectin in the IR region between $4000-400 \mathrm{~cm}^{-1}$. The comparison of FTIR spectra between commercial apple pectin and crude coffee pectin using boiling extraction method is shown in Figure 2. The peak at $1774 \mathrm{~cm}^{-1}$ corresponds to esterified carboxyl groups related to the degree of esterification, while the one at $1668 \mathrm{~cm}^{-1}$ indicates free carboxyl groups [17]. In this study, the coffee pectins from all extraction methods (Figure 3) present strong absorbance at free carboxyl groups and low esterified carboxyl group, indicating low methoxyl pectin. This result is also concurrent with the $\% \mathrm{DE}$ in section 2.1.1. 


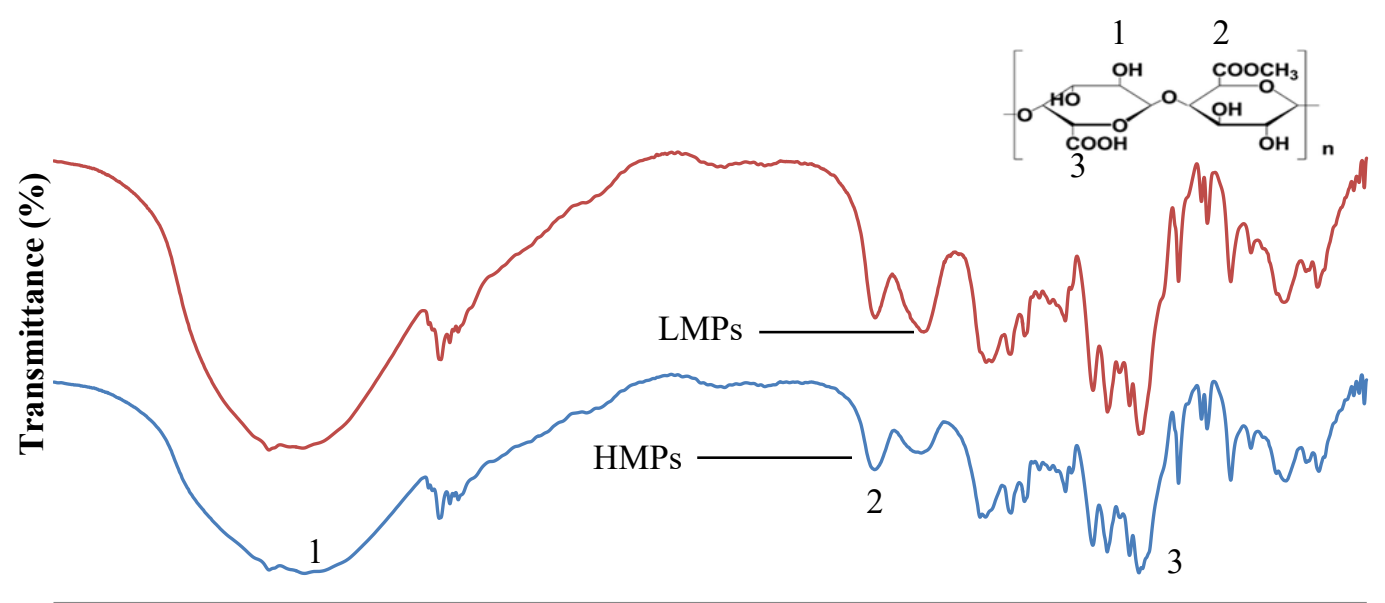

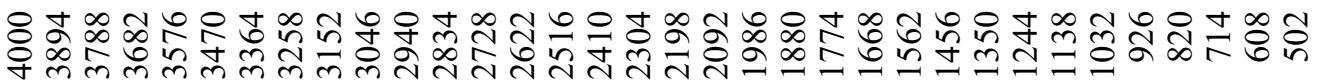

\section{Wavenumbers (cm-1)}

commercial pectin $\quad$ Crude extract

Figure 2 FTIR spectra of coffee pectin extracted by using boiling compared with commercial pectin.

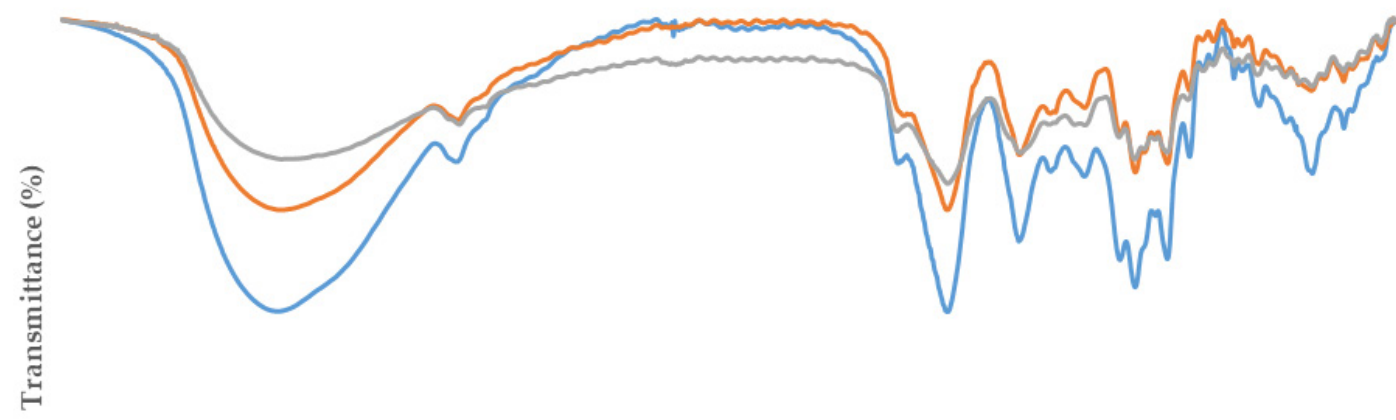

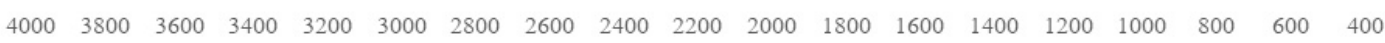

Wavenumber $\left(\mathrm{cm}^{-1}\right)$

- Autoclave Microwave $\longrightarrow$ Boiling

Figure 3 Comparison of FTIR spectra of coffee pectin extracted yield using different extraction methods.

Since coffee pectins from all extraction methods shared similar characteristics, further experiments were conducted using pectin from the boiling extraction method. This pectin also showed the highest yield and could easily be upscaled for large-scale extraction in the future.

Thermo Gravimetric Analysis (TGA)

Figure 4 presents the non-isothermal Thermo Gravimetric Analysis (TGA) and the corresponding Derivative Thermo Gravimetric (DTG) curves of pectin extracted from the coffee pulp and measured in the temperature range of $25-1400{ }^{\circ} \mathrm{C}$. 


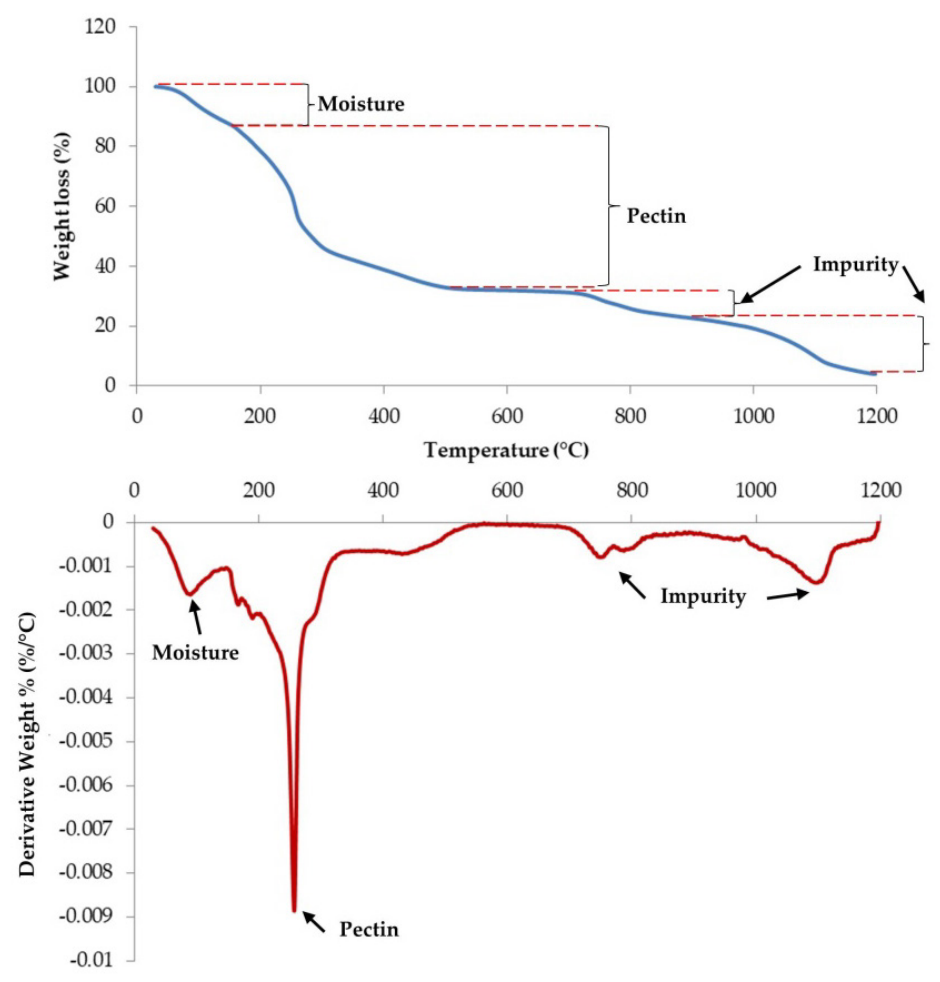

Figure 4 TGA and DTG curves of pectin extracted from coffee pulp.

According to the TGA curve, the thermal decomposition of pectin extracted from the coffee pulp consisted of 4 steps, represented by 4 separate peaks, and observed in the DTG curve. The first decomposition step occurred in the range of $25-150{ }^{\circ} \mathrm{C}$ corresponding to the evaporation of moisture contained in the pectin, which is approximately $20 \% \mathrm{wt}$. The second stage occurred at $150-500{ }^{\circ} \mathrm{C}$, which is the decomposition temperature of pectin counting for $60 \% \mathrm{wt}$. Therefore, this step presents major weights loss. Peaks between $600-900$ and $900-1200{ }^{\circ} \mathrm{C}$ correspond to impurity components from the coffee pulp. At the end of decomposition, the residue remaining is less than $10 \% \mathrm{wt}$.

\section{Determination of the minimum inhibition concentration (MIC) of crude coffee pectin}

The growth curve results of Staphylococcus aureus TISTR 1466 treated with different concentrations of commercial apple pectin and coffee pectin are shown in Figures $\mathbf{5}$ and 6, respectively. Results indicated that commercial pectin, classified as HMP, exhibits insufficient antimicrobial activity at every concentration used in this study. Coffee pectin, which is classified as LMP, displayed some antimicrobial activity with the inhibition concentration in the range of 2.5 to $0.62 \mathrm{mg}$. Nevertheless, antimicrobial activity was only evident in the first $4 \mathrm{~h}$, but did not last for $12 \mathrm{~h}$. The results also showed clearly that at lower concentrations, coffee pectin did not exhibit any antimicrobial activity. This information was used as a guideline for preparing antibacterial films from coffee pectin in the next step. 


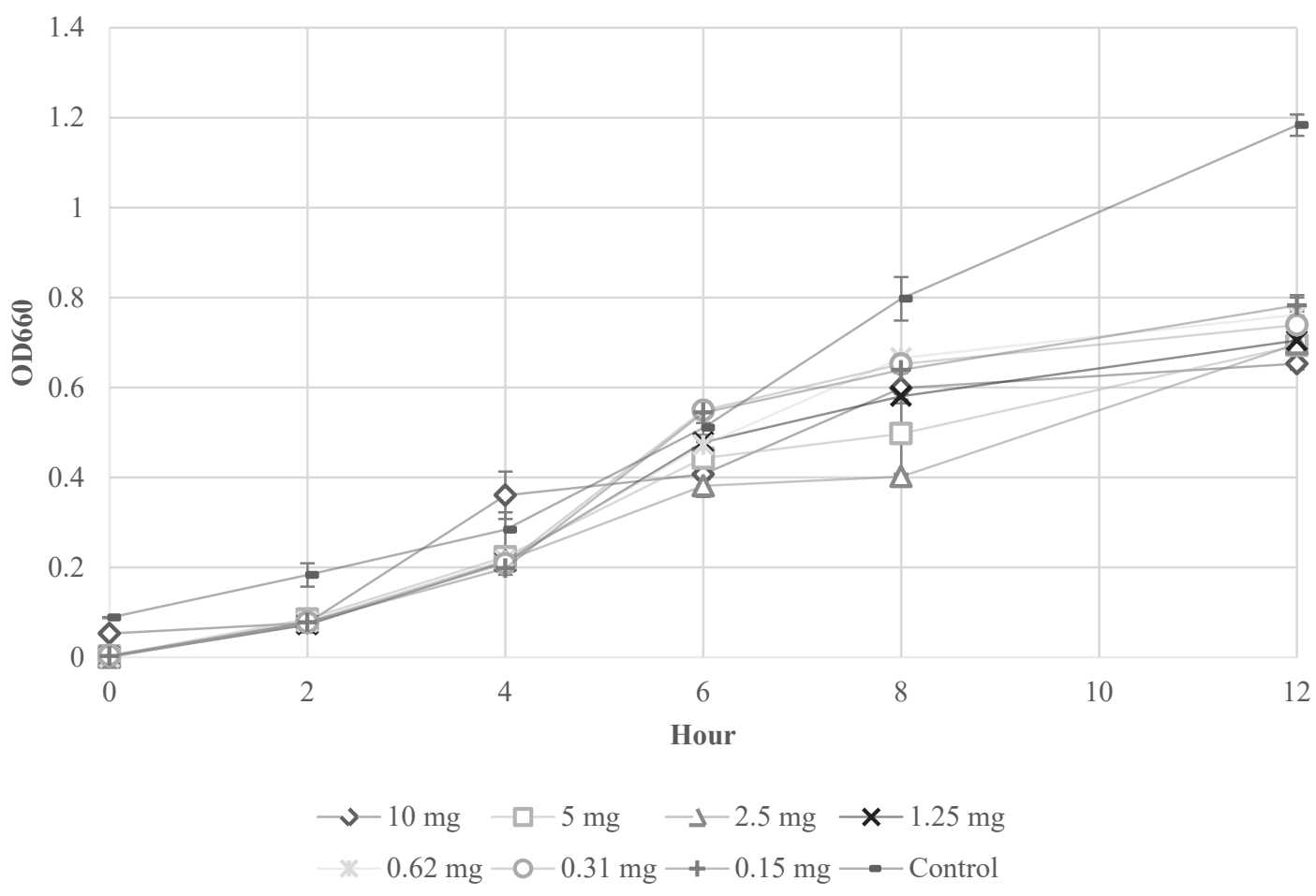

Figure 5 Growth curves of $S$. aureus TISTR 1466 treated with commercial apple pectin at different concentrations for $12 \mathrm{~h}$. Blank Mueller-Hinton Broth was used as control.

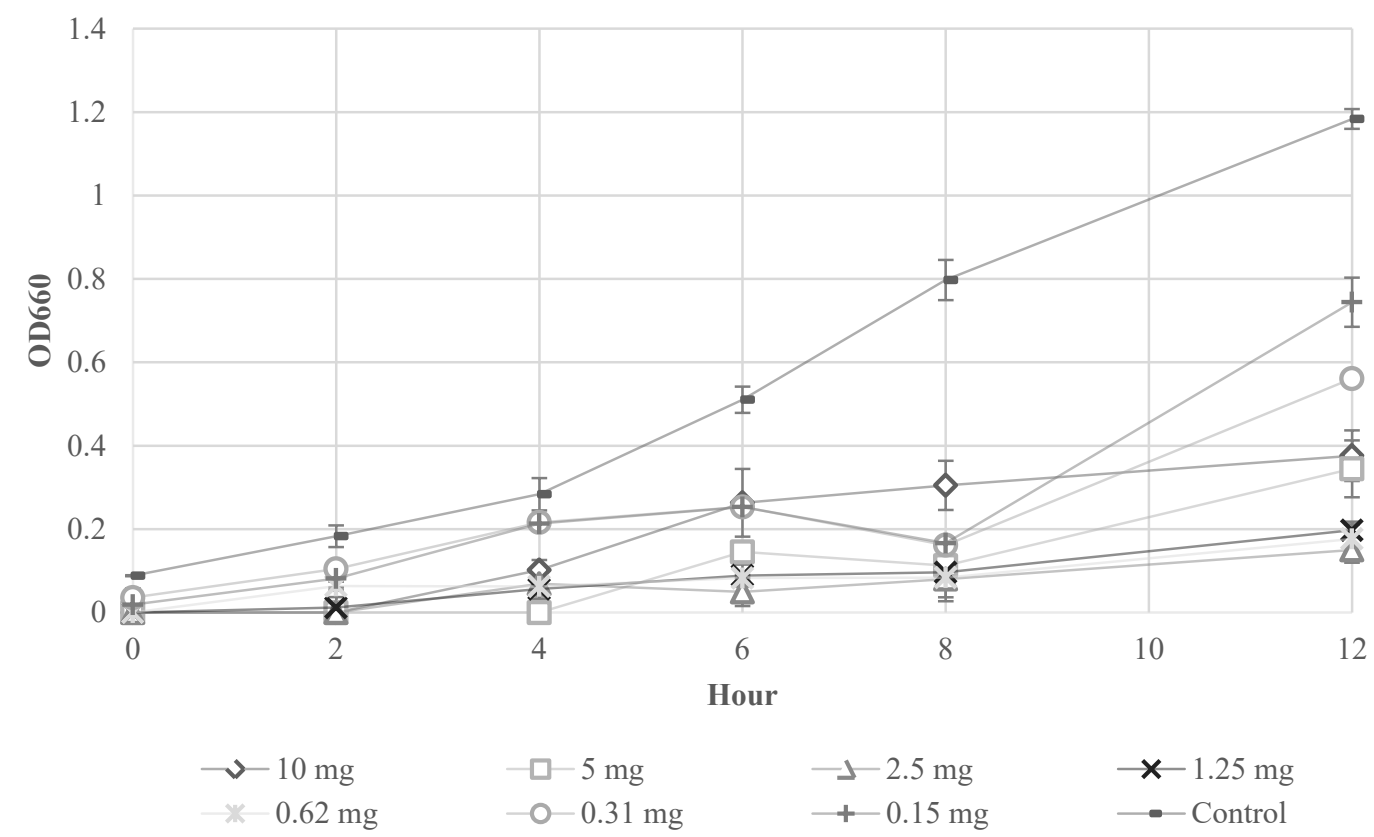

Figure 6 Growth curves of $S$. aureus TISTR 1466 treated with coffee pectin at different concentrations for $12 \mathrm{~h}$. Blank Mueller-Hinton Broth was used as control.

\section{Antibacterial properties of coffee pectin film}

Coffee pectin at $0,1,3$ and $5 \% \mathrm{w} / \mathrm{w}$ concentrations were used for film preparation as described in section 4.4. The prepared films were cut into a disc of $7 \mathrm{~mm}$ in diameter to test for antimicrobial activity 
against Staphylococcus aureus TISTR 1466. A Gentamicin (10 $\mu \mathrm{g})$ susceptibility disc was used as the positive control. Commercial apple pectin or HMP did not exhibit antibacterial activities against $S$. aureus TISTR 1466. The coffee pectin showed antimicrobial inhibition at a concentration of 3 and $5 \%(\mathrm{w} / \mathrm{v})$. Nevertheless, there is no significant difference in antibacterial activity between these 2 concentrations. This result agrees with the MIC results in the previous section. This result agrees with the MIC results in the previous section. Even though the antimicrobial of coffee pectin was not as effective as Gentamicin $(10 \mu \mathrm{g})$, as shown in Table 2, it would be a promising alternative ingredient for film preparation.

Table 2 Antibacterial activity of coffee pectin in various concentrations against S. aureus TISTR 1466.

\begin{tabular}{ccc}
\hline Samples & $\begin{array}{c}\text { Coffee pectin in sample } \\
(\mathbf{m g})\end{array}$ & $\begin{array}{c}\text { Zone of inhibition } \\
(\mathbf{m m})\end{array}$ \\
\hline Coffee pectin 0 \% (Commercial apple pectin) & - & N/A \\
Coffee pectin 1\% & 0.5 & $13.67 \pm 0.58^{\mathrm{a}}$ \\
Coffee pectin 3\% & 1.5 & $19.33 \pm 1.15^{\mathrm{b}}$ \\
Coffee pectin 5\% & 2.5 & $20.67 \pm 0.58^{\mathrm{b}}$ \\
Gentamicin & - & $25.33 \pm 0.58^{\mathrm{c}}$ \\
\hline
\end{tabular}

Noted: Significant level at $95 \%$ confidence $(p<0.05)$.

The present study has shown that the coffee pulp from Arabica coffee (Coffea arabica var. Catimor) is a promising source of low methoxyl pectin. Unlike the antimicrobial properties of pectin from the previous study, which used pectin as a carrier for antimicrobial agents such as silver nanoparticles or essential oils $[8,9,18,19]$, this type of pectin can be used in the biomedical or pharmaceutical fields without structure modification. The boiling extraction method seems to be the most appropriate for largescale extraction, as it yielded the highest amount of pectin. FTIR spectra and \%DE showed that all coffee pectin samples were classified as low methoxyl pectins (LMPs), suggesting potential application in the pharmaceutical industry. Coffee pectin also exhibits antibacterial activity against $S$. aureus TISTR 1466 strain at the range of $0.62-2.5 \mathrm{mg}$ and is suitable for use as an antibacterial film ingredient. Nevertheless, even the higher concentration of pectin did not exhibit sufficient antimicrobial activity. This might be due to pectin forming gel at high concentrations, and the active antimicrobial components could not be released [20]. Pectin from the coffee pulp is a promising alternative material for wound dressing film, especially for oral ulcers, as it is dissolvable. Besides LMPs, the coffee pulp also contains other active compounds such as caffeine, four classes of polyphenols, including flavan-3-ols, hydroxycinnamic acids, flavanols, and anthocyanidins [21-23]. These compounds could cross link with LMPs during film preparation [7]. Further optimization of pectin film formation and drug encapsulation using coffee pectins as a matrix is a promising experiment and could provide additional value to coffee by-products.

\section{Conclusions}

Pectin derived from Arabica coffee pulp (Coffea arabica var. Catimor) belongs to the low methoxyl type. The coffee pectin at concentration range of $2.5-0.62 \mathrm{mg}$ showed potent antibiotic activities close to the Gentamicin standard. Given its antibacterial activities, as well as, the ability to form film, coffee pectin could be potentially used for application in biomedical or pharmaceutical fields. Importantly, pectin from the coffee pulp could add more value to coffee by-products

\section{Acknowledgements}

This research was funded by Mae Fah Luang University research fund, grant number "611B01021". The authors would like to express their sincere gratitude to Akha Coffee Co. Ltd for coffee pulps supplied, Scientific and Technological Instruments Center, Mae Fah Luang University and Thailand Institute of Scientific and Technological Research for Staphylococcus aureus TISTR 1466 strain. Lastly, the authors would like to thanks Dr. Eleni Gentekaki for English correction and improvement. 


\section{References}

[1] Office of Agriculture Economics. Coffee production in Thailand. Annual report, 2019.

[2] U Hasanah, M Setyowati, Edwarsyah, R Efendi, E Safitri, R Idroes, LY Heng and ND Sani. Isolation of pectin from coffee pulp Arabica Gayo for the development of matrices membrane. IOP Conf. Ser. Mater. Sci. Eng. 2019; 523, 012014.

[3] R García, D Arriola, MCD Arriola, ED Porres and C Rolz. Characterization of coffee pectin. $L W T$ Food Sci. Tech. 1991; 24, 125-9.

[4] P Esquivel and VM Jiménez. Functional properties of coffee and coffee by-products. Food Res. Int. 2012; 46, 488-95.

[5] LR Adetunji, A Adekunle, V Orsat and V Raghavan. Advances in the pectin production process using novel extraction techniques: A review. Food Hydrocolloid. 2017; 62, 239-50.

[6] CLO Petkowicz, LC Vriesmann and PA Williams. Pectins from food waste: Extraction, characterization and properties of watermelon rind pectin. Food Hydrocolloid. 2017; 65, 57-67.

[7] C Lara-Espinoza, E Carvajal-Millán, R Balandrán-Quintana, Y López-Franco and A Rascón-Chu. Pectin and pectin-based composite materials: Beyond food texture. Molecules (Basel, Switzerland) 2018; 23, 942 .

[8] L Trejo-González, AI Rodríguez-Hernández, MDR López-Cuellar, VM Juárez-Martínez and N Chavarría-Hernández. Antimicrobial pectin-gellan films: Effects on three foodborne pathogens in a meat medium, and selected physical-mechanical properties. CyTA J. Food. 2018; 16, 469-76.

[9] ST Minzanova, VF Mironov, DM Arkhipova, AV Khabibullina, LG Mironova, YM Zakirova and VA Milyukov. Biological activity and pharmacological application of pectic polysaccharides: A review. Polymers (Basel) 2018; 10, 1407.

[10] Z Daoud, M Sura and R Abdel-Massih. Pectin shows antibacterial activity against Helicobacter pylori. Adv. Biosci. Biotechnol. 2013; 4, 273-7.

[11] W Rakitikul and P Nimmanpipug. Degree of esterification and gelling properties of pectin structure in coffee pulp. Key Eng. Mater. 2016; 675-676, 11-14.

[12] E Calce, V Bugatti, V Vittoria and SD Luca. Solvent-free synthesis of modified pectin compounds promoted by microwave irradiation. Molecules (Basel, Switzerland) 2012; 17, 12234-42.

[13] F Dranca and M Oroian. Optimization of pectin enzymatic extraction from malus domestica 'Fălticeni' apple pomace with celluclast 1.5L. Molecules 2019; 24, 2158.

[14] M Balouiri, M Sadiki and SK Ibnsouda. Methods for in vitro evaluating antimicrobial activity: A review. J. Pharmaceut. Anal. 2016; 6, 71-9.

[15] E Roser, C Grundemann, I Engels and R Huber. Antibacterial in vitro effects of preparations from anthroposophical medicine. BMC Complement. Altern. Med. 2016; 16, 372.

[16] J Hudzicki. Kirby-bauer disk diffusion susceptibility test protocol. American Society for Microbiology, Washington DC, 2009.

[17] MDF Sato, DC Rigoni, MHG Canteri, CLDO Petkowicz, A Nogueira and G Wosiacki. Chemical and instrumental characterization of pectin from dried pomace of eleven apple cultivars. Acta Sci. Agron. 2011; 33, 383-9.

[18] R Ciriminna, A Fidalgo, F Meneguzzo, A Presentato, A Scurria, D Nuzzo, R Alduina, LM Ilharco and M Pagliaro. Pectin: A long-neglected broad-spectrum antibacterial. ChemMedChem 2020; 15, 2228-35.

[19] CG Otoni, MRD Moura, FA Aouada, GP Camilloto, RS Cruz, MV Lorevice, NDFF Soares and LHC Mattoso. Antimicrobial and physical-mechanical properties of pectin/papaya puree/cinnamaldehyde nanoemulsion edible composite films. Food Hydrocolloid. 2014; 41, 188-94.

[20] EG Andriotis, GK Eleftheriadis, C Karavasili and DG Fatouros. Development of bio-active patches based on pectin for the treatment of ulcers and wounds using 3d-bioprinting technology. Pharmaceutics 2020; 12, 56.

[21] A Belščak-Cvitanović and D Komes. Extraction and formulation of bioactive compounds. In: CM Galanakis (Ed.). Handbook of coffee processing by-products. Academic Press, London, 2017, p. 93140 .

[22] BM Dorsey, MA Jones. Healthy components of coffee processing by-products. In: CM Galanakis (Ed.). Handbook of coffee processing by-products. Academic Press, London, 2017, p. 27-62.

[23] MA Ramirez-Coronel, N Marnet, VS Kolli, S Roussos, S Guyot and C Augur. Characterization and estimation of proanthocyanidins and other phenolics in coffee pulp (coffea arabica) by thiolysishigh-performance liquid chromatography. J. Agr. Food Chem. 2004; 52, 1344-9. 